\title{
Minube: Caso de Éxito de una Comunidad Virtual de Viajeros en España
} Minube: A Successful Case of a Travel Virtual Community in Spain

\author{
Ana Belén Bastidas Manzano \\ Universidad a Distancia de Madrid (UDIMA) \\ anabelen.bastidas@udima.es \\ Luis Alberto Casado Aranda \\ Universidad a Distancia de Madrid (UDIMA) \\ luisalberto.casado@udima.es \\ Antonio Rodríguez Ruibal \\ Universidad a Distancia de Madrid (UDIMA) \\ antonio.rodriguez@udima.es \\ Santiago Hernández Hernández \\ Universidad a Distancia de Madrid (UDIMA) \\ santiago.hernandez@cef.es \\ Recibido/Received: 15-02-2019 \\ Aceptado/Accepted: 28-02-2019
}

Este trabajo ha recibido el apoyo del Grupo de Investigación (GI-19/3) Nuevas tendencias en comunicación y marketing (NewteCom)

\section{RESUMEN:}

El sector turístico en España representa el 11,7\% del Producto Interior Bruto, siendo referente en la creación de empleo y posicionándose como un sector estratégico para la creación de empresas. El desarrollo de la tecnología asociada al auge de Internet ha propiciado el fomento de empresas que desarrollan su trabajo en el mundo "online". Así mismo, el turista ha cambiado la forma en la que adquiere sus servicios: participa de lleno en la creación del producto turístico mejorando asî su experiencia turística. Tras un análisis de la evolución tecnológica experimentada por el sector turístico, este trabajo se centra en el concepto de boca-oído electrónico como pieza clave para el posicionamiento de empresas/destinos turísticos. Posteriormente, explora cómo la comunidad virtual de viajeros minube, pequeña empresa española que supone una manifestación de ese boca-oído gracias a la Web 2.0, ha mejorado la experiencia turística de los viajeros.

Palabras clave: turismo; Web 2.0; boca-oído; boca-oído electrónico; comunidad virtual de viajeros; usuario generador contenido

\section{ABSTRACT:}

The tourism sector in Spain accounts for $11.7 \%$ of the GDP, thus being crucial for the job creation and business expansion. The development of technology linked to the Internet growth has helped the creation of online firms. Likewise, the tourist has now changed the way to purchase services: (s)he participates widely in the creation of the touristic product, hence improving his/her 
touristic experience. After an analysis of the technological evolution experienced by the tourism sector, the current research focuses on the concept of electronic word of mouth (e-wom) as a key piece for the positioning of tourism destinations/firms. Then, the paper explores how the virtual community minube, a small Spanish business developed in the Web 2.0 environment, has substantially improved the tourism experience of travelers.

Keywords: tourism, Web 2.0, wom, e-wom, virtual communities, Travel 2.0, user generation content (UGC)

\section{CÓMO CITAR ESTE ARTÍCULO/HOW TO CITE THIS ARTICLE}

Bastidas Manzano, A.B.; Casado Aranda, L.A.; Rodríguez Ruibal, A.; Hernández Hernández, S. (2019): Minube: Caso de Éxito de una Comunidad Virtual de Viajeros en España. Rotur. Revista de Ocio y Turismo, 13(1): 12-27. https://doi.org/10.17979/rotur.2019.13.1.5008

\section{INTRODUCCIÓN}

El sector turístico supone un sector clave para España, representando el 11,7\% del PIB, 2,7 millones de empleos y el 12,2\% de afiliación a la Seguridad Social (Hoteltur, 2019). España recibió en 2018 82,6 millones de turistas internacionales, con un aumento interanual del gasto turístico de un 3\%, lo que supuso un gasto total de 90.000 millones de euros (IET, 2018). La Figura 1 revelan, además, que España ocupa una posición predominante en el ranking de países más visitados anualmente. A su vez, destacan las ganancias por turismo en España, que se sitúa en segundo lugar a escala internacional (Figura 2).

Figura 1. Países más visitados y con más ganancias en turismo en 2017. Adaptado de Organización Mundial del Turismo, UNWTO (2018).

\section{(\#) LOSPISESDEL MUNDOQUE UNWTO}

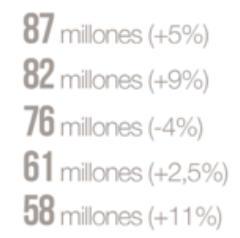

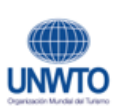

\section{LOSPAISES DEL MUNDOQUEMÁS GANAN CONEL TURISMO२०17}

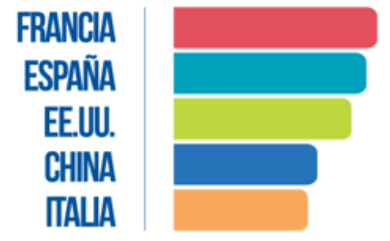

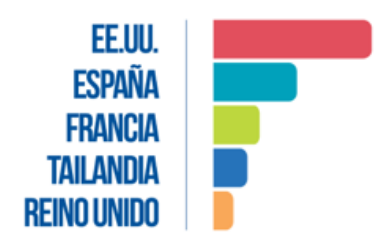

Una de las características estructurales de la economía española es el déficit que tiene lugar entre las diferencias de las exportaciones y las importaciones. Este es en parte subsanado por el buen comportamiento de la balanza de pagos del sector turístico. El superávit de la partida de turismo y viajes supera desde 1998 los 20.000 millones de euros, y en concreto, en 2018 supuso 36.787 millones de euros (Banco de España - Balanza de Pagos y el IET, 2018). Los datos extraídos del IET muestran, además, que el $80 \%$ de los turistas que visitan España son usuarios de Internet, seis de cada diez búsquedas en la red están relacionadas con viajes y destinos, y el $40 \%$ del comercio electrónico corresponde a la venta de productos turísticos. 
Figura 2. Peso del turismo de la economía nacional. El peso del turismo en la economía nacional

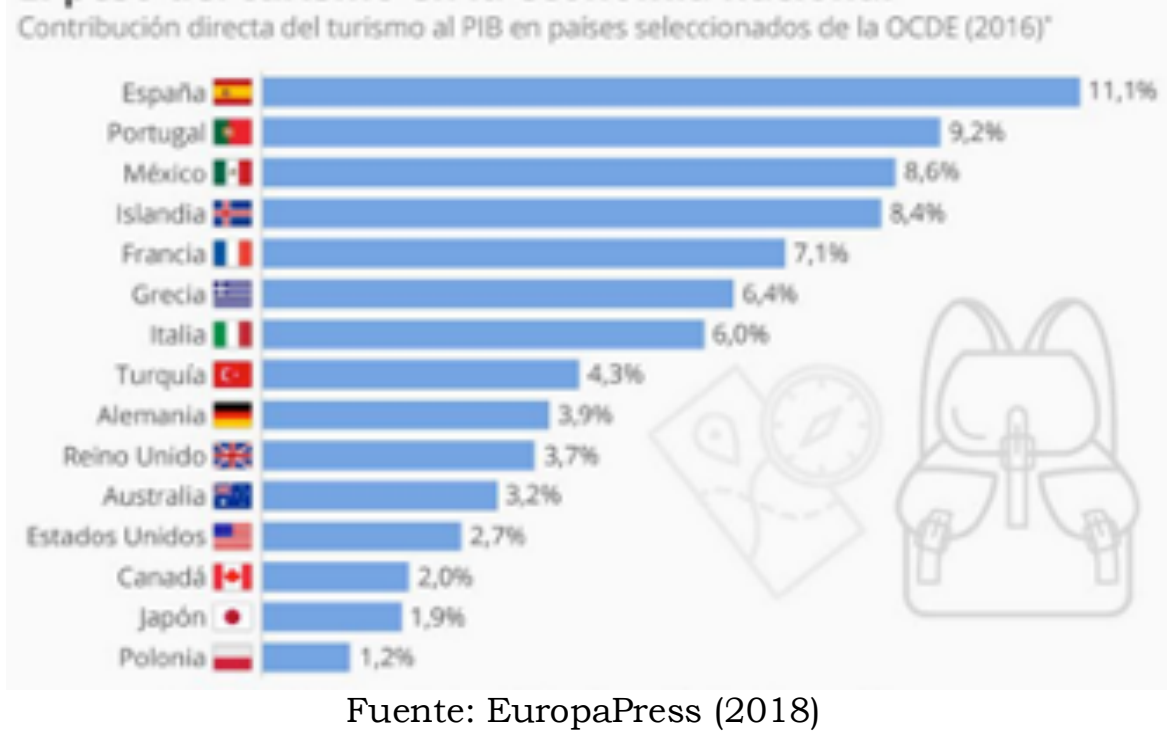

Al hilo de este desarrollo económico, el turismo ha ido adaptándose a las necesidades del consumidor para conseguir atraer al turista. Concretamente, la industria turística ha sido pionera en el uso de las tecnologías de la información y la comunicación, principalmente en la distribución de los servicios turísticos. Los Sistemas Globales de Distribución utilizada por los touroperadores en los años 80 han dado paso a las Tecnologías de la Información y Comunicación (TIC), que han potenciado el papel del proveedor, quien puede obtener información directa de sus clientes, así como la opción de vender directamente a ellos, evitando por consiguiente la comisión procedente de la transacción con intermediarios (Law et al., 2004). Se ha pasado de una etapa en la que el acceso a los viajes era propiedad de las rentas altas, a la actualidad, cuando es el cliente quien prepara su viaje, acude directamente a los proveedores de transporte y alojamiento, y consulta previamente las opiniones de otros viajeros.

La relación entre tecnología y turismo ha sido ampliamente estudiada (Berné Manero, GarcíaGonzález, García-Uceda, \& Múgica Grijalba, 2012; Brown \& Chalmers, 2003). El uso de las TIC en turismo presenta varios beneficios, tanto para las empresas como para los turistas. En concreto, algunos de los beneficios para el consumidor/turista son los siguientes: (Pradhan, Oh, y Lee 2018)

(i) Mejora el proceso de búsqueda: ofreciendo por tanto mejores y mayores opciones al consumidor.

(ii) Acceso a los mejores precios y opciones de viaje: la oferta es más amplia y el acceso a la misma es más rápido y sencillo.

(iii) Ayuda a hacer la experiencia turística más fascinante y agradable: imágenes, vídeos, experiencias de otros viajeros, incentivan tanto el pre-viaje como el pos-viaje.

(iv) Disminuye el "riesgo" del viaje (por el hecho de comprar un producto intangible). Gracias a tecnologías como la realidad virtual, el viajero obtiene la máxima información sobre un destino y/o empresa de forma previa al viaje, haciéndose una imagen previa mental sobre las expectativas de la experiencia. La tecnología ayudará a que esa imagen "inventada" sea lo más cercana a la realidad.

Según la revisión del trabajo realizado por Berne, Garcia-Gonzalez, \& Mugica, (2012), los principales cambios que el uso intensivo de las TIC en el sector servicios está provocando, se deben a: 
Cambios en el establecimiento y el desarrollado de relaciones entre los agentes implicados. Existe una tendencia clara por eliminar de la cadena de distribución el papel del intermediario, como está sucediendo en las aerolineas aéreas (Gonzalez Santamaria 2009). Siendo éstas mismas las que comercializan sus billetes a través de sus propias Web llegando directamente al consumidor final, sin necesidad de intermediarios.

(ii) Cambios en las diferentes posiciones de poder de los agentes. Los cambios en la cadena de valor van a depender del uso que el consumidor haga de los servicios ofrecidos.

(iii) Cambios en la política de producto. Servicios integrados con interdependencia entre ellos, así como la creación de servicios turísticos personalizados en función de la demanda del consumidor.

(iv) Cambios en cómo el consumidor participa en los procesos. Para la industria turística la participación en los procesos de compra por parte del consumidor es cada vez mayor, siendo creciente incluso la participación en la creación de los propios productos turísticos. Hablamos de productos personalizados o a la carta. Es el usuario el creador de contenido y el que demanda participar de manera más directa en la propia creación del producto turístico.

Estos cambios tecnológicos afectan al tradicional "boca-oído" (WOM, por sus siglas en inglés, Word-of-mouth), que repercuten a su vez en la imagen de la empresa/destino turístico. Westbrook (1987) define el WOM como "la comunicación informal entre los consumidores sobre las caracteristicas, propiedad y uso de un producto o servicio, en el que las fuentes se consideran independientes de la influencia comercial". El desarrollo de Internet ha dado lugar al e-WOM (electronic word of mouth). Hennig-Thurau et al. (2006) lo definieron como "cualquier declaración positiva o negativa creada por clientes potenciales, actuales o antiguos acerca de un producto, servicio o empresa, y que se ofrece a una multitud de personas e instituciones a través de Internet". Los consumidores de viajes buscan otras opiniones para gestionar el riesgo percibido de un producto intangible. La literatura previa, de hecho, apoya la influencia del boca oreja y la recomendación de los clientes en el establecimiento de comercio electrónico (Smith, Menon, y Sivakumar 2005). A su vez, la popularidad de redes sociales como Facebook, Instagram, LinkedIn y Twitter ha crecido exponencialmente en los últimos años, hecho que está provocando cambios en efectos del e-WOM. El WOM no se lleva a cabo ya en el contexto exclusivo del cara a cara, que implica una fuente y un destinatario (Vilpponen, Winter, y Sundqvist 2006; Kozinets et al. 2010). Más bien, se produce en incremento del dominio público de una forma más transparente a través de las redes sociales online (Ho y Dempsey 2010).

Reconociendo esta tendencia, los empresarios del sector han comenzado a aprovechar las redes sociales con el fin de generar conciencia, interés y, finalmente, la compra del producto. Su estrategia general consiste en la creación de páginas relacionadas con el producto, y luego tratan de atraer clientes potenciales a las páginas través de la publicidad y e-WOM (Green y Dougherty 2008). En el ámbito turístico, se ha demostrado que los hoteles que han tenido buenos comentarios online han aumentado el número de sus reservas (Velázquez, Blasco, y Saura 2015), convirtiendo de esta manera al e-WOM en una herramienta clave del marketing. De esta forma, el e-WOM ha resultado ser una de las más importantes fuentes de información a la hora de planear un viaje (López y Sicilia 2014)

Este contexto ha propiciado el auge de las comunidades virtuales online, que ofrecen una oportunidad a los turistas para buscar información de viajes, establecer relaciones con otros y, de esta forma, tomar la decisión sobre el viaje, conociendo siempre los precios en los distintos puntos de venta (Arsal et al. 2010). A pesar del crecimiento experimentado por este tipo de comunidades virtuales, el estudio de sus repercusiones sobre la experiencia del turista apenas ha sido analizado, y resultaría clave: (i) porque el crecimiento de las comunidades virtuales es 
paralelo a la rápida propagación del fenómeno de la innovación, a través del aumento de la conectividad entre los agentes implicados en las actividades de innovación (Jeppesen y Frederiksen 2006), (ii) por la relevancia de la innovación para impulsar el desarrollo del turismo (Weiermair 2006) y, (iii) por el impacto creciente de Internet en el turismo (Alonso y Blasco 2006). Esta investigación precisamente analiza el éxito y relevancia de la comunidad virtual de origen español minube, así como sus repercusiones sobre la experiencia del turista. Minube es una start-up española, creada en el año 2007 por ingenieros y que destaca por su proyección de futuro y fuerte ritmo de crecimiento. Es referente para los blogueros de viajes, realizando cada año la "Quedada de Viajeros en FITUR", posee gran repercusión en el sector de los viajes y un gran impacto en redes sociales.

\section{REVISIÓN DE LA LITERATURA}

\section{II.I. Enfoque tradicional del turismo Vs Turismo actualidad}

La influencia de las TIC en el turismo no es nueva. A partir de la década de 1970, las TIC han propiciado importantes cambios en la actividad turística, con la evolución de las centrales de reserva (CRS) a los sistemas globales de distribución (GDS) y, a finales de los años noventa, con la aparición de Internet (Buhalis y Law, 2008), hasta el punto de que Sheldon (1997) identificó la información del turismo, y las TIC, como la columna vertebral de las operaciones turísticas. Los destinos turísticos, por su parte, desarrollan sistemas propios para mejorar su representación, impulsar su imagen y atrae reservas directas.

Para entender el desarrollo tecnológico en el sector turístico debemos hablar, en primer lugar, del desarrollo de los transportes en la década de los 60, que propició el conocido "turismo de masas" (Valls, 1996). Posteriormente, se sucedieron tres etapas fundamentales. La primera, en la década de 1970, con el nacimiento de los Sistemas Informatizados de Reservas (central distribution system, CRS); la segunda, en 1980 con el surgimiento de los Sistemas Globales de Distribución (global distribution system, GDS); y, por último, en los 90, con el desarrollo de Internet.

- Los CRS se caracterizaron por (Buhalis 1998): (i) se desarrollan en la década de los 70, (ii) son fundamentales para la distribución y estrategia de las aerolíneas, (iii) se trata de bases de datos que administran un enorme inventario de datos turísticos, (iv) las aerolíneas fueron pioneras en su uso, seguidas posteriormente de cadenas hoteleras y operadores turísticos, (v) mejoran la rentabilidad de los procesos por la reducción de costes, (vi) contribuyen a la gestión estratégica de la industria turística, (vii) los CRS suelen cobrar una comisión muy competitiva en comparación con otras opciones de distribución, a la vez que ofrece la opción de precios flexibles para adaptarse a la oferta y demanda, y (viii) reducen costes de comunicación, mientras que proporciona información sobre los patrones de demanda, así como la posición de socios y competidores.

- Por su parte, los GDS destacan por (Buhalis 1998): (i) ser una evolución de los CRS de las aerolíneas, ampliando gradualmente su cobertura geográfica, mediante la integración tanto horizontal (con otros sistemas de líneas aéreas) como vertical (incorporando toda la gama de productos turísticos y servicios tales como alojamiento, alquiler de automóviles, billetes de tren y ferry, entretenimiento, etc,.), (ii) surgen en la década de los 80 y siguen vigentes en la actualidad, (iii) la integración de los CRS con los GDS permitió la compra de la mayor parte de las empresas de la industria turística, (iv) conectan a la mayoría de las organizaciones de turismo con intermediarios de todo el mundo, (v) ofrecen cada vez más productos de ocio y de negocios, proporcionando información y permitiendo reservas para entradas de teatro, paquetes vacacionales y destinos turísticos, (vi) con la llegada de Internet los GDS están creando plataformas que les permita atraer también a los 
consumidores a través de metaWebs que permitan la reserva de servicios turísticos, aumentando de esta manera su productividad y eficiencia, y, (vii) los GDS se están aprovechando de las ventajas de Internet para ofrecer interfaces innovadoras que mejoren la comunicación directa con los consumidores, mejorando las oportunidades de venta.

Figura 3. Evolución de la tecnología en turismo

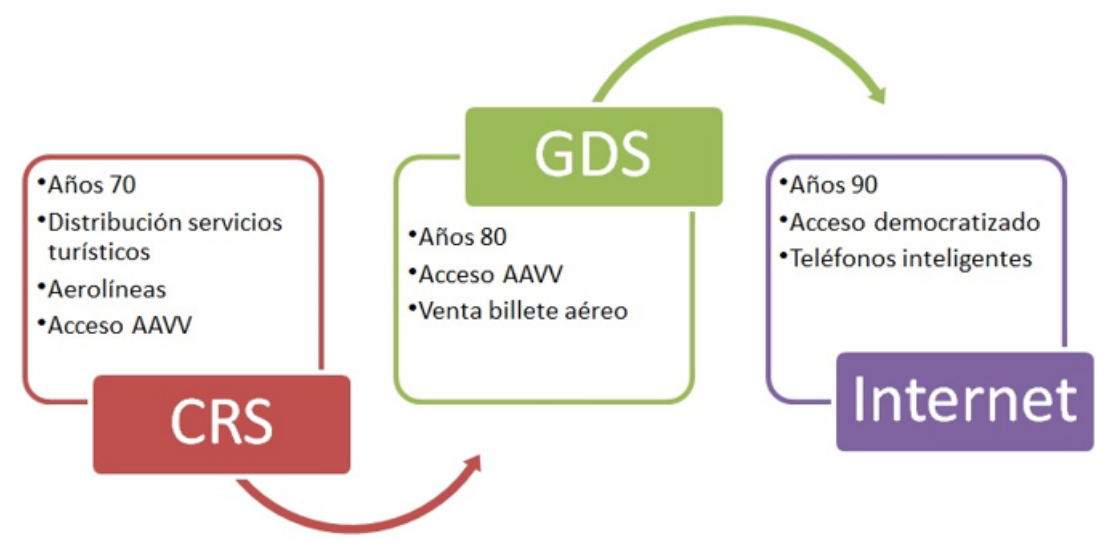

- Internet, ya más recientemente, ha cambiado el papel de los intermediarios de turismo y, especialmente, las agencias de viajes. La coincidencia de las agencias de viajes tradicionales con el desarrollo de la Red supone un reto para el sector. Esto también se ve reforzado por la reciente limitación de la comisión por parte de las aerolíneas en todo el mundo. Es por ello, que el desarrollo de Internet en los años 90 revolucionó el sector servicios, sobre todo en la manera de llegar al consumidor final. La democratización en cuanto el acceso libre a la Red por parte del usuario final, ha cambiado radicalmente las reglas del juego. Surgen grandes oportunidades en la comercialización del sector servicios gracias al uso de las nuevas tecnologías, ya que que estás han influenciado en la manera de seleccionar un destino, hacer la reserva y comentar tras el viaje (Sánchez Jiménez, Fernández Allés, y Mier-Terán Franco 2018). La información es un aspecto fundamental del turismo. El sector necesita nutrirse de datos para el desarrollo de su función. Tanto previa a la actividad turística en sí, como durante su desarrollo y posterior valoración tras el viaje, el consumidor turístico demanda información en todo el proceso de la experiencia. La competitividad del sector es cada día mayor, ofreciendo, en muchos casos, una oferta muy similar. El uso de la tecnología de la información y la comunicación en todo el proceso, conseguirá proporcionar una ventaja competitiva para las empresas y destinos de cara a un mercado turístico globalizado.

\section{II.II. El caso concreto de Internet en turismo}

Las TIC han transformado el mercado de las comunicaciones y, en especial, el sector turístico. En los últimos años, Internet se ha convertido en el canal principal para la búsqueda y propagación de información (Law, Qi, y Buhalis 2010). El desarrollo de las TIC y, particularmente de Internet, da lugar a un nuevo turista, que dispone de mucha información y demanda un valor excepcional del tiempo y el dinero. Como consecuencia, la comercialización y promoción de los servicios turísticos ha cambiado radicalmente en los últimos años. Según Arroyo (2016) el turismo electrónico reúne a todas las tecnologías que se aplican en sector turístico, reflejando a su vez la digitalización de los procesos y las cadenas de valor en los viajes. 
Para que la relación entre Internet y turismo se establezca de forma adecuada, es fundamental que la Web, que es el primer lugar donde accede el usuario en búsqueda de información turística (y que forma parte de la experiencia), cumpla las siguientes características:

- Usabilidad: "La medida en que un producto puede ser usado por determinados usuarios para conseguir objetivos específicos con efectividad, eficiencia y satisfacción en un contexto de uso específico " (ISO 9241-11, 2011)

- Diseño Web (arquitectura de la información): Es decir, que la información en la Web siga un orden correcto y fácil de encontrar.

- Encontrabilidad: No se puede usar lo que no se encuentra. Es fundamental la aparición en redes sociales, blog, estrategia de marketing (a través del proceso de búsqueda en buscadores, la llamada búsqueda orgánica, o a través de la búsqueda patrocinada, desarrollada por la publicidad que las empresas insertan en los motores de búsqueda a través de apuestas para aparecer en los primeros resultados), etc, Estas apariciones favorecen que el usuario encuentre el contenido que demanda.

- Usuario como generador de contenido (UGC): Posibilitar al usuario la opción de añadir opiniones sobre un servicio disfrutado ofrece contenido más fiable a la Web. (Por ejemplo, las comunidades virtuales de viajeros)

- Experiencia de Usuario: Será el objetivo final que se pretenda a través de un diseño Web amigable. Una experiencia de usuario adecuada favorecerá el proceso de compra, y la satisfacción del usuario en relación a sus expectativas (Tussyadiah, 2014).

El desarrollo de Internet y su llegada al consumidor final también ha sido de manera paulatina. Mientras que en un primer momento el acceso a la Red estaba limitado a empresas, usuarios especializados o profesionales del sector, el proceso ha ido cambiando, con tarifas planas más asequibles, velocidad en el envío y recepción de los datos online. Internet se ha posicionado como la mayor fuente de información para los viajeros (Pan, MacLaurin, y Crotts 2007). En este contexto, el desarrollo de las TIC ha proporcionado información y publicidad, siendo la tecnología móvil el medio que usan los turistas por excelencia, tanto para la elección previa del destino, durante sus vacaciones y con posterioridad a éstas, sustituyendo, de esta manera, a las agencias de viajes tradicionales. Para Tussyadiah (2014) el aumento del contenido digital creado por los turistas ha mejorado el conocimiento sobre los destinos y empresas turísticas, así como las imágenes previas creadas de éstos. Hablamos de un nuevo modelo de Internet donde consultar, publicar, discutir, compartir y distribuir información sobre turismo, cuyo contenido sea creado por los usuarios interactuando entre ellos (Gomis 2008).

$\mathrm{El}$ auge de las opiniones de viajeros en sitios Web ha sido fruto del propio desarrollo de la llamada Web 2.0, una Web que permite a los usuarios contribuir al desarrollo, colaboración y distribución del contenido y personalizándolo (Vickery y Wunsch-Vincent 2007). Los consumidores acceden a multitud de información a través de blogs de viajes, canales de vídeo, comunidades virtuales o redes sociales, modificando de esta forma la manera en la que preparan y compran sus vacaciones. Estudios recientes corroboran que los viajeros suelen comentar más en sitios webs de terceros relacionados con viajes, que en las propias webs de la empresa y/o destino (Burgess, Parish, y Alcock 2011). Es fundamental en este contexto mostrar la identidad de la persona que inserta en comentario para mejorar la credibilidad por parte de los usuarios (Kusumasondjaja, Shanka, y Marchegiani 2012) consiguiendo de esta manera que las criticas positivas sean más fiables y los comentarios negativos más creíbles.

El desarrollo de Internet, unido a la actual Web 2.0, ha instaurado un término al que la literatura referente al turismo está haciendo referencia: es el Usuario como Generador de Contenido (UGC). El contenido generado por el usuario hace referencia al contenido creado o producido por el público en general sin ser pagado por profesionales, y normalmente distribuido a través de Internet (Daugherty, Eastin, y Bright 2008). Ejemplos del UGC en la Red son diversos: escribir y participar en blog, leer o escribir críticas de servicios, formar parte en foros o participar en redes sociales (Carrera et al. 2008). Los usuarios desean compartir su experiencia y evaluación en redes sociales después de comprar un producto o servicio, creando de esta manera un flujo 
enorme de e-WOM (Mendes-Filho y Tan 2009). En todas las actividades de UGC, el usuario está en el punto central siendo no solo un consumidor, sino también aportando en Internet (Arrigara y Levina 2008). Como resultado, los usuarios desempeñan un enorme papel en la Web (George y Scerri 2007) generando: análisis y comentarios, entretenimientos, críticas (de libros, restaurantes, productos, comentarios de hoteles). El UGC está cambiando la dinámica de la industria de los viajes valiéndose del enorme e-WOM desarrollado (Arsal et al. 2010; MendesFilho y Tan 2009). Los viajeros se ponen en contacto directamente con otros viajeros que tienen intereses de destino similares a través de las redes sociales en el sector de viajes online.

\section{II.III. Plataformas virtuales: el caso de minube}

El concepto de plataforma virtual surge precisamente en este contexto de e-WOM y UGC. Las plataformas virtuales de viajeros son precisamente espacios digitales que permiten a los usuarios compartir opiniones, leer, comentar, comparar y recomendar (o no) a otros usuarios un destino o producto turístico. Hoy en día todo el sector turístico depende en gran medida de plataformas como Tripadvisor, Booking o del caso español de minube, entre otras. La Tabla 1 recoge las principales plataformas digitales del turista expandidas a nivel internacional que proporcionan información al usuario en el momento de la compra.

Los puntos claves para entender la dependencia a las plataformas virtuales en turismo son (González Jiménez, 2017):

1. Están integradas socialmente en el proceso de decisión de compra de un producto o servicio turístico. El usuario antes de finalizar la compra, lee y valora la opinión de otros usuarios.

2. Las plataformas virtuales permiten la comparación de precios del mismo producto o servicio turístico, y en la mayoría de los casos son comisionistas de las ventas finalizadas por los lectores.

3. El usuario, compre o no el servicio, puede opinar al respecto en la mayoría de los casos.

4. Suelen ser plataformas adaptadas a cualquier soporte (ordenador, tablet o smartphone).

5. Minube ha sido un ejemplo de evolución de comunidad virtual española de éxito. En un principio esta plataforma de lengua hispana, surgió como punto de encuentro de comunidad de viajeros. Con el uso de las nuevas tecnologías en el sector turístico y desde hace pocos meses, esta plataforma permite a los usuarios contar con su propio espacio. Ahora los usuarios no sólo pueden difundir su actividad como viajeros, sino que pueden acceder a la compra de productos y servicios.

Tabla 1. Características de las principales plataformas virtuales de viajeros con incidencia internacional y cotización en Bolsa 


\begin{tabular}{|c|c|c|c|c|c|}
\hline Plataforma & Año & Nacionalidad & Datos cuantitativos & Servicios & Ingresos \\
\hline $\begin{array}{l}\text { (1) } \\
\text { tripadvisor } \\
\text { https: } / / \mathrm{bit} . \mathrm{lv} / 2 \mathrm{cs} 7 \\
\underline{\mathrm{cI}}\end{array}$ & 2000 & Estadounidense & $\begin{array}{l}\text { - Más de } 702 \text { millones de } \\
\text { opiniones y comentarios } \\
-\quad 190 \text { contribuciones por } \\
\text { minuto } \\
\text { - Más de } 10 \text { billones de } \\
\text { contribuciones a nivel mundial } \\
\text { - Más de } 3.000 \text { empleados } \\
\text { - Es la mayor comunidad } \\
\text { virtual de viajeros a nivel } \\
\text { mundial }\end{array}$ & $\begin{array}{l}\text { - Indice de popularidad de los } \\
\text { hoteles } \\
\text { - Vídeos y Fotografias subidos } \\
\text { por los propios usuarios } \\
\text { - Foros para viajeros } \\
\text { - Guía de restaurantes y } \\
\text { alojamientos y de vuelos y } \\
\text { - Búsqueda de } \\
\text { estimación de tarifas aéreas } \\
\text { - Calculadora de alquileres } \\
\text { vacacionales } \\
\text { - Reservas hoteleras }\end{array}$ & $\begin{array}{l}\text { Su modelo de negocio } \\
\text { procede principalmente } \\
\text { (más del } 75 \% \text { de la } \\
\text { publicidad } \\
\text { Más de } 1.500 \text { millones de } \\
\text { dólares en } 2017\end{array}$ \\
\hline $\begin{array}{l}\text { K } \\
\end{array}$ & 2004 & Estadounidense & $\begin{array}{l}\text { - más de dos mil millones de } \\
\text { búsquedas de viajes al año } \\
\text { - más de } 1.000 \text { empleados } \\
\text { - } 12 \text { oficinas internacionales } \\
\text { - más de } 20 \text { idiomas }\end{array}$ & $\begin{array}{l}\text { - Es un metabuscador de } \\
\text { viajes: vuelo, hotel, coche de } \\
\text { alquiler o viaje perfecto. }\end{array}$ & $\begin{array}{l}\text { Su gran fuente de } \\
\text { ingresos procede de la } \\
\text { publicidad }\end{array}$ \\
\hline $\begin{array}{l}\text { https://bit.lv/2MK } \\
\underline{\text { XXiA }}\end{array}$ & 1996 & Holandesa & $\begin{array}{l}\text { - Más de } 1.550 .000 \text { noches de } \\
\text { reservas diarias. } \\
\\
\text { - Más de } 17.000 \text { trabajadores } \\
\text { en } 198 \text { oficinas de } 70 \text { países } \\
\text { de todo el mundo } \\
\text { La web y apps de Booking.com } \\
\text { están disponibles en más de } \\
40 \text { idiomas, y ofrecen } \\
28.344 .343 \text { opciones de } \\
\text { alojamiento en total, en } \\
145.419 \text { destinos de } 228 \\
\text { países y territorios en todo el } \\
\text { mundo. }\end{array}$ & $\begin{array}{l}\text { - Es una agencia de viajes } \\
\text { online } \\
\text { - Oferta amplia de } \\
\text { alojamientos, como } \\
\text { apartamentos, casas, resorts } \\
\text { de lujo, bed \& breakfasts de } \\
\text { gestión familiar y hasta iglús } \\
\text { y casas en los árboles. }\end{array}$ & $\begin{array}{l}\text { Priceline, grupo online } \\
\text { propietario de } \\
\text { Booking.com, ha obtenido } \\
\text { beneficios de } 2.010 \mathrm{M} €, \\
\text { en el año } 2017 \text { un } 16,3 \% \\
\text { menos que en año anterior }\end{array}$ \\
\hline $\begin{array}{c}\text { airbnb } \\
\text { https://bit.ly/2Uqv } \\
\underline{5 x p}\end{array}$ & 2008 & Estadounidense & $\begin{array}{l}\text { - Más de } 5 \text { millones de } \\
\text { anuncios en todo el mundo } \\
\text { - } 81.000 \text { ciudades con } \\
\text { alojamientos de Airbnb } \\
\text { - Más de } 191 \text { países presentes } \\
\text { en Airbnb } \\
\text { - Más de } 2 \text { millones de media } \\
\text { de huéspedes por noche }\end{array}$ & \begin{tabular}{|l|} 
- Es una plataforma de \\
alquiler vacacional \\
- En Airbnb se anuncian más \\
de 3.000 castillos y 2.200 \\
casas en el árbol. \\
- Alquiler vacacional \\
- Alquiler habitaciones en \\
casa compartida \\
- Alquiler apartamento / casa \\
completa
\end{tabular} & $\begin{array}{l}2,6 \text { miles de millones USD } \\
\text { (2017) }\end{array}$ \\
\hline
\end{tabular}

Fuente: Elaboración propia

\section{II.IV. Objetivos de investigación}

$\mathrm{El}$ enorme crecimiento del UGC, unido al auge de las comunidades virtuales de viajeros, hacen necesario identificar cuáles son los factores de éxito de dichas comunidades virtuales y sus repercusiones sobre la experiencia del usuario. A pesar de que la comunidad de viajeros española minube posee dimensiones menores a Tripadvisor o Booking, por ejemplo, continúa siendo una de las plataformas de mayor acceso de turistas españoles y constituye un punto de referencia para la planificación de la experiencia turística (La Razón, 2017), por lo que resulta crucial detectar sus factores de éxito.

\section{METODOLOGÍA}

Siguiendo la línea de estudios previos (Tinsley y Lynch, 2001), la presente investigación pretendió determinar los factores de éxito de minube mediante una entrevista en profundidad semiestructurada, esto es, "el entrevistador tuvo una serie de cuestiones iniciales de interés, que moldeó a medida que avanzó la conversación" (Fielding, 1993). Más concretamente, el entrevistador se reunió con el $\mathrm{CEO}$ de minube con el objetivo de que aportara información útil sobre cuáles, a juicio del CEO, constituyen los principales factores de éxito de la comunidad de 
viajeros española. El Apéndice 1 incluye las cuestiones planteadas durante la entrevista que engloba, entre otros aspectos, referencias a fuentes de ingresos, tipo de contenido de redes sociales, capacidad de réplica de las empresas turísticas en su plataforma o relación con partners de interés.

\section{RESULTADOS}

Minube es una empresa española creada en 2007, QUE incluye en su página web información en español, italiano, portugués, francés e inglés, y está presente en:

- España

- LATAM (foco en México, Argentina, Colombia, Chile y Brasil)

- Sur de Europa (Portugal, Italia, Francia)

- Reino Unido

- USA

\section{IV.I. Productos de la marca}

Minube dispone de un servicio Web y una aplicación. Esta última ha sido elegida por Google e iOS como una de las mejores aplicaciones del 2015. Se trata de la primera y única aplicación Android española que forma parte de Editor's Choice de Google Play, la lista de las mejores aplicaciones existentes. Dicha app incluye guías para viaje y un servicio específico para empresas. La Web minube.com proporciona un índice de popularidad de los hoteles, guías webs, opciones de rutas, mapas, foro, comunidad y blog de usuarios. La app de minube ha sido visitada por más de 30 millones de viajeros en todo el mundo y posee 4 millones de descargas.

IV.II. Minube en cifras

\begin{tabular}{|c|c|}
\hline Caracteristica & Cifras \\
Fotos subidas & 3 millones \\
Puntos de interés internacionales con & 350.000 \\
recomendaciones o experiencias & \\
Recomendaciones de texto de usuarios & 800.000 \\
Usuarios registrados & 2,7 millones \\
Empleados & 28 \\
Porcentaje de contenido compartido por & $70 \%$ \\
usuarios en smartphones & \\
Beneficios 2017 & 1,7 millones de euros \\
Visitas al año & $>95$ millones \\
\hline
\end{tabular}

De importancia destacar que minube implementa tres principales modelos de ingresos: comisiones de hoteles, campañas de marketing para destinos turísticos y marcas relacionadas con viajes y display. En palabras del CEO: "el modelo de futuro, sin embargo, pasa por convertirnos en una OTA (agencia de viajes online) de Tours \& Activities sin perder el foco en el resto de patas de viaje".

\section{IV.III. El CEO opina: puntos fuertes y débiles}

A minube la definen tres valores muy característicos: "crecimiento sostenible, innovación y pasión por el trabajo". Los trabajadores de minube están "comprometidos con el desarrollo de un turismo responsable y sostenible, la innovación y el aprendizaje colaborativo". De hecho, minube es Patrocinador Oficial del Año Internacional del Turismo Sostenible para el Desarrollo 2017 en 
alianza con la OMT. Fruto de esta visión general, se desprenden los principales puntos fuertes y débiles:

\section{Puntos fuertes}

- Creación de guías offline para el viajero

- Quedada de viajeros

- La app se puede descargar sin conexión a Internet

- Elaboración de vídeos de promoción turística para empresas y destinos

- Creación de un Observatorio Turístico donde se publican informes sobre el perfil del turista, los cambios en el sector y evolución del turismo inteligente

- Colaboración con instituciones públicas turísticas para la publicación conjunta de guías y vídeos.

- Sus contenidos se pueden compartir y comentar en Facfebook y Twitter.

- La página web y app son totalmente intuitivas, atractivas y fáciles de usar.

- Existe "Minube School", una iniciativa c-learning de minube para co-crear entre todos una industria turística responsable y sostenible, para crear un mundo mejor. Se trata de un punto de encuentro c-learning para el diálogo, el empowermente y la búsqueda de la excelencia.

- Su objetivo es generar aprendizaje colaborativo online y offline, entre los diferentes stakeholders del sector turístico.

\section{Puntos débiles}

- Todavía es una Red relativamente pequeña en comparación con TripAdvisor.

- No ofrece la opción de réplica por parte del empresario

- Cierto desconocimiento por parte del turista.

- No se ofrecen opiniones de alojamiento

- Aunque apuestan sólo por comentarios positivos en la Web también hay críticas sobre ciertos servicios.

El análisis de los datos disponibles sobre la empresa española de minube desprende que estamos ante una pequeña empresa (por su número de trabajadores, no más de 30) pero con una gran capacidad de negocio. Minube apuesta por la tecnología y el turismo digital. Ha entendido muy claramente el concepto 2.0 y hace del usuario el centro de su contenido. A su vez, la expansión del negocio hacia otras áreas como "minube School" y su apuesta por un turismo sostenible hace que estén siempre a la vanguardia, luchando por mejorar y adaptarse a las necesidades del mercado. La empresa ha cambiado varias veces el modelo de negocio, aunque en líneas generales sigue trabajando por lo mismo: una plataforma basada en los consejos de viajeros.

\section{DISCUSIÓN Y CONCLUSIONES}

La contribución directa del turismo al PIB en España es la mayor de todos los países de la OCDE (2016), aportando el 11,1\% a dicha magnitud. El auge de las TIC ha tenido un efecto directo sobre la forma de gestionar los productos turísticos por parte de las organizaciones, pero también para los usuarios. Las TIC, de hecho, han mejorado el proceso de búsqueda de servicios turísticos, el acceso a los mejores precios, ha ayudado a potenciar la experiencia del turista y ha disminuido el riesgo del viaje. El auge de la Web 2.0 ha situado al turista como un usuario generador de contenido que busca, opina, comparte, compara y recomienda destinos turísticos en base a su experiencia.

Este contexto ha propiciado el auge de las comunidades virtuales online, que ofrecen una oportunidad a los turistas para buscar información de viajes, establecer relaciones con otros y, de esta forma, tomar la decisión sobre el viaje, conociendo siempre los precios en los distintos puntos de venta (Arsal et al. 2010). A pesar del crecimiento experimentado por este tipo de comunidades virtuales, el estudio de sus repercusiones sobre la experiencia del turista apenas 
ha sido analizado (Alonso y Blasco 2006). Esta investigación precisamente explora el éxito y relevancia de la comunidad virtual de origen español minube, así como sus repercusiones sobre la experiencia del turista. Mediante una entrevista en profundidad semiestructurada al CEO de minube, este trabajo repasa los productos de dicha plataforma virtual y los puntos fuertes $\mathrm{y}$ débiles que la ayudan a tener éxito en nuestro país.

La start-up española creada en el año 2007 se sitúa en una posición inferior respecto a empresas como Kayak, TripAdvisor o AirBnB en número de trabajadores, opiniones, descargas o cifras de ingresos. No obstante, ha conseguido instaurarse como una de las plataformas virtuales para turistas españoles de referencia. Los resultados muestran que tres puntos clave pueden definir el éxito de minube: (i) cantidad y calidad de los servicios que ofrece, que iguala incluso supera en diversidad de servicios a la competencia: índice de popularidad de los hoteles, guías webs, opciones de rutas, mapas, foro, comunidad y blog de usuarios, pero también cuenta con "Minube School", iniciativa c-learning que fomenta el diálogo, empowerment y búsqueda de la excelencia; (ii) gran adaptación a la digitalización y Web 2.0, creando una app intuitiva, atractiva y fácil de usar, que incluso fue elegida por Google e iOS como una de las mejores aplicaciones del 2015; y (iii) cultura organizativa orientada al éxito, ecologia y aprendizaje colaborativo, idiosincrasias con gran potencial de futuro (Jacob y Pérez, 2008). Se diferencia, además, del resto de plataformas virtuales su triple modelo de negocio: comisiones de hoteles, campañas de marketing para destinos turísticos y marcas relacionadas con viajes y display. El resto de plataformas utilizan la publicidad como principal vía de ingresos.

Si bien minube tiene aún importantes retos de futuro, como incrementar la reputación e imagen de marca, incorporar nuevos servicios de réplica a comentarios por parte del empresario u ofrecer opiniones de alojamientos, su apuesta por conceptos clave de futuro, como la sostenibilidad, digitalización y web colaborativa, podrían ser clave para su éxito. De hecho, como plataforma virtual supone en sí una tipología más de economía colaborativa. Las nuevas formas de entendimiento de la economía actual, ha hecho que se hable de un concepto más global en donde el ciudadano puede participar de manera más activa ya no sólo como consumidor, sino como proveedor de servicios, compartiendo por ejemplo sus pertenencias como un coche o una casa. Es la llamada economía colaborativa, que tiene como ejemplos más conocidos Uber, Cabify o Blablacar. Su futuro más por aspectos legales que por funcionamiento, plantea un escenario incierto, pero el modelo de negocio no deja de ser impecable en cuanto a las cifras que manejan. En esta línea, la consultora PWC estimaba en 2016 que el mercado para negocios de economía colaborativa ofrecía una oportunidad potencial de 335.000 millones de dólares para 2025. El $55 \%$ de la población española ha usado, además, al menos una vez una plataforma colaborativa en 2016 (Eastwind, 2017), razón más que suficiente para augurar un futuro lleno de éxito para empresas que apuestan por la economía colaborativa, como minube.

\section{BIBLIOGRAFÍA}

Alonso, M., y Elies Furio B. (2006). "Internet y sus aplicaciones al sector turístico". https://halshs.archives-ouvertes.fr/halshs-00119439/document.

Arrigara, M., y Levina, N. (2008). "Social Dynamics in Online Cultural Fields". ICIS 2008 Proceedings, enero. https://aisel.aisnet.org/icis2008/120.

Arsal, I., Woosnam K., Baldwin E., y Backman S. (2010). "Residents as Travel Destination Information Providers: An Online Community Perspective". Journal of Travel Research 49 (4): 400-413. https://doi.org/10.1177/0047287509346856.

Berné Manero, C., García-González, M., García-Uceda E., y Múgica Grijalba J.M. (2012). "Modelización de los cambios en el sistema de distribución del sector turístico debidos a la incorporación de las tecnologías". Cuadernos de Economía y Dirección de la Empresa 15 (3): 117-29. https://doi.org/10.1016/j.cede.2011.07.002. 
Berné Manero, C., García-González, M., García-Uceda E., y Múgica Grijalba J.M. (2013). "Identificación y análisis de los criterios de cambios del sistema de distribución turístico asociados al uso intensivo de las tecnologías de la información y de la comunicación". Investigaciones Europeas de Dirección y Economía de la Empresa 19 (2): 90-101. https://doi.org/10.1016/j.iedee.2012.09.002.

Barry B, y Chalmers M. (2003). “Tourism and Mobile Technology". En ECSCW 2003, editado por Kari Kuutti, Eija Helena Karsten, Geraldine Fitzpatrick, Paul Dourish, y Kjeld Schmidt, 335-54. Springer Netherlands.

Buhalis, D, (1998). "Strategic use of information technologies in the tourism industry". Tourism Management 19 (5): 409-21. https://doi.org/10.1016/S0261-5177(98)00038-7.

Burgess, L., Parish B., y Alcock C. (20119. "To What Extent Are Regional Tourism Organisations (RTOs) in Australia Leveraging the Benefits of Web Technology for Destination Marketing and ECommerce?" Electronic Commerce Research 11 (3): 341-55. https://doi.org/10.1007/s10660-011-9077-1.

Carrera, P., Chiu, C., Pratipwattanawong P., Chienwattanasuk S., Fatimah Syed Ahmad S., y Murphy J. (2008). "MySpace, My Friends, My Customers". En Information and Communication Technologies in Tourism 2008, editado por Peter O'Connor, Wolfram Höpken, y Ulrike Gretzel, 94-105. Springer Vienna.

Daugherty, T., S. Eastin M., y Bright L. (2008). "Exploring Consumer Motivations for Creating User-Generated Content". Journal of Interactive Advertising 8 (2): 16-25. https://doi.org/10.1080/15252019.2008.10722139.

Eastwind (2017). La economía colaborativa española en cifras. (2017, June 16). Retrieved January 31, 2019, from https://eastwind.es/saringmadrid-2017-la-economiacolaborativa-espanola-cifras/

EuropaPress (2018). El peso del turismo. (2018, Diciembre 18). Retrieved January 31, 19, https://www.europapress.es/turismo/nacional/noticia-peso-turismo-117-pib-128empleo-espana-2017-20181218122856.html

George, C. E., y Scerri J. (2007). "Web 2.0 and User-Generated Content: Legal Challenges in the New Frontier". SSRN Scholarly Paper ID 1290715. Rochester, NY: Social Science Research Network. https://papers.ssrn.com/abstract=1290715.

Gomis Filgueira, J.M. (2008). "LOS BLOGS DENTRO DEL TURISMO 2.0". ROTUR: Revista de Ocio y Turismo 1 (1): 135-52. https://doi.org/10.17979/rotur.2008.1.1.1230.

González Jiménez, P.M. (2017). "La oferta de viviendas de uso turístico a través de plataformas colaborativas: reflexiones desde el derecho de la competencia desleal”, Revista de Estudios Europeos, 70: 111-126.

Green, G.P., y Dougherty M. /2008). Localizing Linkages for Food and Tourism: Culinary Tourism as a Community Development Strategy". Community Development 39 (3): 148-58. https://doi.org/10.1080/15575330809489674.

Hennig-Thurau, T., Groth M., y Gremler D. (2006). "Are All Smiles Created Equal? How Emotional Contagion and Emotional Labor Affect Service Relationships”. Journal of Marketing 70 (3): 58-73. https://doi.org/10.1509/jmkg.70.3.58. 
Ho, J.., y Dempsey M. (2010). "Viral marketing: Motivations to forward online content". Journal of Business Research, Advances in Internet Consumer Behavior\& Marketing Strategy, 63 (9): 1000-1006. https://doi.org/10.1016/j.jbusres.2008.08.010.

Instituto de Estudios Turísticos (2018) Datos económicos sector turístico. Disponible en:https://www.tourspain.es/enus/ConocimientoTuristico/Pages /EmpleoTuristico/Afil iacionSS/InformesPublicaciones.aspx (acceso 30 Enero 2019).

Jacob, M. y Pérez, E. (2008). "La innovación en el sector turístico: El caso de Baleares". Rotur 1:51-64.

Jeppesen, L.B., y Frederiksen L. (2006). "Why Do Users Contribute to Firm-Hosted User Communities? The Case of Computer-Controlled Music Instruments". Organization Science 17 (1): 45-63. https://doi.org/10.1287/orsc.1050.0156.

Kozinets, R.V., de Valck K., Wojnicki A., y J.S Wilner S. (2010). "Networked Narratives: Understanding Word-of-Mouth Marketing in Online Communities". Journal of Marketing 74 (2): 71-89. https://doi.org/10.1509/jmkg.74.2.71.

Kusumasondjaja, S., Shanka T., y Marchegiani C. (2012). "Credibility of Online Reviews and Initial Trust: The Roles of Reviewer's Identity and Review Valence". Journal of Vacation Marketing 18 (3): 185-95. https://doi.org/10.1177/1356766712449365.

La Razón (2017). Minube, la startup donde empiezan los viajes. Disponible en: https://www.larazon.es/economia/minube-la-start-up-donde-empiezan-los-viajesAD15071100 (acceso 30 Enero 2019).

Law, R., Qi S., y Buhalis D. (2010). "Progress in tourism management: A review of website evaluation in tourism research". Tourism Management 31 (3): 297-313. https://doi.org/10.1016/j.tourman.2009.11.007.

Law, Rob, RJames Wong, y Kenith Leung (2004). "The impact of the Internet on travel agencies". International Journal of Contemporary Hospitality Management 16 (2): 100-107. https://doi.org/10.1108/09596110410519982.

López, M., y Sicilia M. (2014). "Determinants of E-WOM Influence: The Role of Consumers\&rsquo; Internet Experience". Journal of theoretical and applied electronic commerce research 9 (1): 28-43. https://doi.org/10.4067/S071818762014000100004.

Mendes-Filho, L., y Tan F. (2009). "User-generated content and consumer empowerment in the travel industry: a uses \& gratifications and dual-process conceptualization". PACIS 2009 Proceedings, julio. https://aisel.aisnet.org/pacis2009/28.

Organización Mundial del Turismo (2018). Compendio de datos turísticos. Disponible en: http://statistics.unwto.org/es/content/datos (acceso 30 Enero 2019)

Pan, B., MacLaurin T., y Crotts J. (2007). "Travel Blogs and the Implications for Destination Marketing". Journal of Travel Research 46 (1): 35-45. https://doi.org/10.1177/0047287507302378. 
Pradhan, M.K., Oh J., y Lee H. (2018). “Understanding Travelers' Behavior for Sustainable Smart Tourism: A Technology Readiness Perspective". Sustainability 10 (11): 4259. https://doi.org/10.3390/su10114259.

Sánchez Jiménez, M.A., Fernández Allés, M.T., y Mier-Terán Franco, J.J. (2018). "Revisión teórica de la relevancia de las nuevas tecnologias de la comunicación (TIC) en el sector turístico". TURYDES Revista Turismo y Desarrollo local sostenible, n. ${ }^{\circ} \quad$ junio (junio). $\quad$ https://www.eumed.net/rev/turydes/24/tecnologia-turismo.html.

Saranow, J. (2004). "Getting Travel Advice From a Stranger; Online Blogs Emerge As Popular Resource; How to Find Useful Ones". The Wall Street Journal, 28(3).

Smith, D., Menon S., y Sivakumar, K. (2005). "Online Peer and Editorial Recommendations, Trust, and Choice in Virtual Markets". Journal of Interactive Marketing 19 (3): 15-37. https://doi.org/10.1002/dir.20041.

Tinsley, R., y Lynch, P. (2001): Small tourism business networks and destination development. International Journal of Hospitality Management, 20(4), 367-378.

Tussyadiah, I.P. (2014). "Toward a Theoretical Foundation for Experience Design in Tourism". Journal of Travel Research 53 (5): 543-64. https://doi.org/10.1177/0047287513513172.

Velázquez Moliner, B., Fuentes Blasco M., y Gil Saura I. (2015). "ICT Adoption in Hotels and Electronic Word-of-Mouth/La Adopción de La TIC En Hoteles y El Boca-Oreja Electrónico". Academia; Bogotá 28 (2): 227-50. http://dx.doi.org/10.1108/ARLA-102013-0164.

Vickery, G., y Wunsch-Vincent, S. (2007). Participative Web And User-Created Content: Web 2.0 Wikis and Social Networking. Paris, France, France: Organization for Economic Cooperation and Development (OECD).

Vilpponen, A., Winter S., y Sundqvist S. (2006). "Electronic Word-of-Mouth in Online Environments". Journal of Interactive Advertising 6 (2): 8-77. https://doi.org/10.1080/15252019.2006.10722120.

Weiermair, K. (2006). "Prospects for Innovation in Tourism". Journal of Quality Assurance in Hospitality \& Tourism 6 (3-4): 59-72. https://doi.org/10.1300/J162v06n03 04.

Westbrook, R. A. (1987). "Product/Consumption-Based Affective Responses and Postpurchase Processes”. JMR, Journal of Marketing Research; Chicago 24 (3): 258. 


\section{ANEXO 1}

1. ¿Cómo surgió la idea de la creación de la empresa?

2. ¿Cuál fue el capital inicial? ¿Quiénes son los socios actuales? ¿Recibís algún tipo de ayuda pública? En definitiva, ¿cuál es vuestro modelo de negocio o fuentes de ingresos?

3. Breve evolución histórica de minube desde su creación a la actualidad

4. ¿Nos podéis ofrecer algún dato sobre la reputación de minube en comparación con otras empresas del sector?

5. ¿Tenéis alguna alianza con algún grupo turístico?

6. Importancia de las redes sociales para el incremento de usuarios, ¿cómo ha sido la evolución de usuarios de la Web a través del tiempo? ¿A qué es debido?

7. Perfil de los empleados de minube

8. Puntos fuertes y débiles de minube

9. Tendencias futuras comunidades virtuales de viajeros, ¿hacia dónde irá el sector? ¿hacia dónde quiere dirigirse minube? 